\title{
Proceedings of the 25th International Stroke Genetics Consortium Workshop: Cambridge, UK
}

Neurol Genet 2020;6:e421. doi:10.1212/NXG.0000000000000421

\section{Proceedings of the 25th International Stroke Genetics Consortium Workshop, April 10-12, 2019, Cambridge, UK}

Jemma C. Hopewell, $P h D^{1}$, Matthew Traylor, $P h D^{2}$, and Hugh S. Markus, FMedSci ${ }^{2}$

${ }^{1}$ CTSU, Nuffield Department of Population Health, University of Oxford; and ${ }^{2}$ Department of Clinical Neurosciences, University of Cambridge, United Kingdom.

The 25th workshop of the International Stroke Genetics Consortium (ISGC) was held at the beautiful and historic Queens' College, University of Cambridge, UK on April 10-12, 2019, and was hosted by Associate Professor Jemma C Hopewell, Dr Matthew Traylor, and Professor Hugh Markus, and co-hosted by Professor Jin-Moo Lee and Associate Professor Carlos Cruchaga. Herein, we present the Proceedings and official published abstracts of the 25th ISGC Workshop.

The ISGC is an international collaboration of physicians and scientists who have agreed to pool resources and expertise in an effort to unravel the genetic basis of stroke and its comorbidities. Founded in 2007 by a small group of stroke genetics investigators, the ISGC has grown to over 200 members representing over 50 countries in North and South America, Europe, Australia, Africa, and Asia. ISGC workshops are held semi-annually and provide a forum for ISGC investigators to report on progress of ongoing scientific projects and discuss new ideas that help advance the ISGC's research mission. The ISGC nurtures its junior members and many of the peer-reviewed abstracts in these Proceedings reflect the contributions of active junior investigators. Reflecting this priority, in addition to the travel awards made available by the ISGC, this meeting benefitted from funding from the British Heart Foundation in order to support an unprecedented number of junior investigators to attend and present their work. We encourage you to visit our website (http://www.strokegenetics.org) for further details.

Presentations at the workshop included invited lectures, ISGC working group reports, and short presentations about ongoing research projects and new collaborative proposals. The meeting focused on major translational areas reflecting the theme of "Genes to Therapies in the Big Data Era" and the current international research priorities across both the ISGC and the global stroke genetics research community. Invited international experts included Professor Sir Rory Collins, the highly acclaimed Principal Investigator and Chief Executive of UK Biobank, Professor Nicole Soranzo, a celebrated expert in functional genomics, Dr John Whittaker, an industry leader in identifying the causal genes and mechanisms to inform drug discovery, as well as others. This meeting also marked the presentation of the inaugural Michele Sale award for Women in Stroke Genetics, an internationally competitive mid-career award dedicated to the memory of Dr Michele Sale, which was awarded to Associate Professor Jemma Hopewell. We are particularly grateful to Anna Bates for her excellent administrative support for the meeting.

On behalf of the ISGC Steering Committee and its many members, we hope that you enjoy reviewing these Proceedings.

Full author information, including institutions, disclosures, and contact information, can be found at: links.lww.com/NXG/A258. 
Acknowledgment: Steering Committee-Jin-Moo Lee, Chair and Israel Fernandez-Cadenas, Co-Chair; Members: Stephanie Debette (Immediate Past-Chair), Ann-Katrin Giese (Junior member); Rufus Akinyemi, Jemma Hopewell, Steven Kittner, Jane Maguire, Paul Nyquist, Natalia Rost. Working Group Leaders-Acute Endophenotypes WG: Israel FernandezCadenas, Jin-Moo Lee; Cognitive WG: Matt Pase, Brad Worrall; Imaging WG: Natalia Rost; Intracranial Aneurysm WG: Ynte Ruigrok, Philippe Bijlenga; Intracerebral Hemorrhage WG: Guido Falcone, Jonathan Rosand, Dan Woo; META/MEGASTROKE WG: Stephanie Debette, Martin Dichgans, Jemma Hopewell; Multiomics WG: Carlos Cruchaga, Myriam Fornage; Neuro-CHARGE WG: Myriam Fornage, Sudha Seshadri; SiGN WG: Steven Kittner, Brackie Mitchel; Translational Science WG: Chris Anderson, Tom Van Agtmael. Administrative support: Bailey Montgomery, Anna Bates.

Correspondence Hugh S. Markus hsm32@cam.ac.uk

\section{Periventricular and subcortical} white matter hyperintensities are associated with different vascular risk factors

M.D. Schirmer, $P h D^{1,2,3}$, M.J. Nardin, $M D^{1}$, and F. Dubost, $\mathrm{MSc}^{4}$; on behalf of the MRI-GENIE Consortium

Objective To determine whether there are differences in risk factors for periventricular ( $\mathrm{pWMH}$ ) and subcortical (sWMH) white matter hyperintensity (WMH) burden. Background WMH burden is a critically important cerebrovascular phenotype linked to the prognosis of acute ischemic stroke (AIS) and can be differentiated into sWMH and pWMH. Design/ Methods We utilized the MRI-GENetics Interface Exploration (MRI-GENIE) study, a large-scale, international, hospital-based collaborative study of AIS patients with clinical Fluid Attenuated Inversion Recovery (FLAIR) images available. Stroke etiology was assessed using the TOAST classification system (cardioembolic [CE], small artery occlusion [SAO], large artery atherosclerosis [LAA], Other, and Undetermined). WMH and ventricles were segmented with deep-learning algorithms, designed for clinical FLAIR images. Utilizing both segmentations, we differentiated between pWMH and sWMH, where sWMH is comprised of disconnected lesions, not directly adjacent to the ventricles. We analyzed effects of risk factors (age, sex, hypertension, diabetes, smoking and TOAST subtypes [excluding Undetermined]) using uni- and multivariate linear regression models for sWMH and pWMH. The site of acquisition was included as a nuisance parameter in all models. Results Successful automated segmentation of WMH and ventricles were available in 3959 patients. Three thousand four hundred sixty patients had full phenotypic information available and were (mean \pm standard deviation) $59 \pm 16$ years old, $60 \%$ male, $63 \%$ hypertensive, $21 \%$ diabetic, and $43 \%$ with history of smoking (ever/never). Stroke subtypes were CE (23\%), LAA (17\%), SAO (25\%), Other (7\%) and Undetermined (28\%). Model parameters are summarized in table 1. Age, hypertensive and smoking status modified sWMH and pWMH burden. Furthermore, sex and SAO were also associated with pWMH. Conclusions In conclusion, risk factors affect sWMH and pWMH, where sex and SAO only affect the latter.

Contact Email: MSCHIRMER1@mgh.harvard.edu

\section{Introduction to Helsinki ischemic and hemorrhagic stroke GWAS cohorts}

Liisa Tomppo, $M D, P h D^{1}$, Hanne Sallinen, $M D^{1}$, Priit Palta, $P h D^{2}$, Javier Nuñez Fontarnau, $P h D^{2}$, Aki S. Havulinna, DSc (Tech. $)^{2,3}$, Samuli Ripatti, $P h D^{2}$, Veikko Salomaa, $M D, P h D^{3}$, Turgut Tatlisumak, $M D, P h D^{4,5}$, and Daniel Strbian, $M D, P h D^{1}$

Objective To collect extensively phenotyped study samples to study genetic background of ischemic stroke (IS) and intracerebral hemorrhage (ICH), stroke outcome and related phenotypes in the Finnish population. Background Helsinki 2000 Ischemic Stroke Genetics Study and Helsinki Intracerebral Hemorrhage Genetics Study include IS and ICH cases respectively recruited at the Department of Neurology, Helsinki University Hospital, which is the only neurological emergency service with a catchment area of 1.6 million inhabitants. Design/Methods Neuroimaging-verified IS cases have been recruited between April 2012 and December 2017 and ICH cases since February 2014 (ongoing). Detailed phenotype data including etiologic classification (TOAST for IS and SMASH-U for ICH), neuroimaging and laboratory results, NIHSS on admission and for IS cases also at 24 hours, main cardiovascular comorbidities and previous cardiovascular events, medication details and life style risk factors have been collected from patients' electronic medical charts. The patients are followed up at 3 months and at 1-year and mRS is obtained at these time points. The control samples are participants of the National FINRISK Study, which is a large cross-sectional population study with standardized health examinations and linkage to the national health registries. All the samples are genotyped with Illumina genome-wide genotyping chips. For genotype imputation, Finnish SISu (Sequencing Initiative Suomi) whole-genome sequencing reference panel of 3775 individuals and $16.9 \mathrm{M}$ variants on chromosomes 1-22 and X is used. Results The current datasets include 1650 IS cases (age $65.0 \pm 14.4$ years, $41.4 \%$ females, TOAST: $20.5 \%$ large artery stroke, $32.0 \%$ cardioembolic stroke, $9.6 \%$ small vessel stroke, $37.9 \%$ other or undetermined cause), $494 \mathrm{ICH}$ cases (age $66.3 \pm 13.5$ years, $42.9 \%$ females, SMASH-U: $38.5 \%$ hypertensive angiopathy, $21.1 \%$ amyloid angiopathy, $15.8 \%$ medication, $24.7 \%$ other or undetermined cause), and 3340 stroke-free controls (age 61.4 


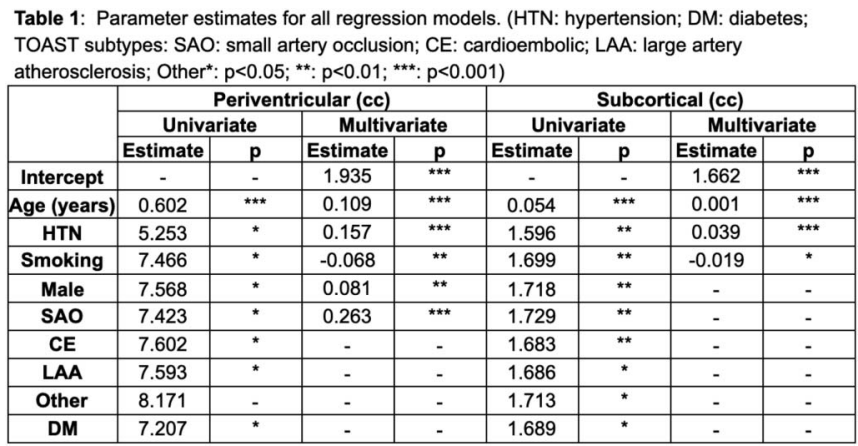

\pm 13.8 years, $55.8 \%$ females) after genotype and phenotype data quality control checks. Imputation yields $14.4 \mathrm{M}$ variants with $\mathrm{INFO}>0.4$ out of which $8.8 \mathrm{M}$ variants have MAF $>0.01$. Conclusions This ongoing study and genotyped samples provide a comprehensive dataset for genetic analyses of stroke in Finland and the data can be combined with international study cohorts.

Contact Email: Liisa-Maaria.Tomppo@hus.fi

\section{Exome array analysis of early-onset ischemic stroke}

John W. Cole, $M D, M S^{1,2}$, Thomas Jaworek, BS $S^{1}$ Kathy Ryan, $M S^{1}$, Brady Gaynor, $B S^{1}$, Patrick McArdle, $P h D^{1}$, Oscar C. Stine, $\mathrm{PhD}^{1}$, Hugo J. Aparicio, MD, MPH ${ }^{3}$, Yan Gao, $\mathrm{MPH}^{4}$, Xiaochen Lin, $P h D^{5}$, Megan L. Groves, $P h D^{6}$, Steven J. Kittner, $M D, M P H^{1,2}$, James Wilson, $M D^{4}$, Qiong Yang, $P h D^{3}$, Sudha Seshadri, $M D^{3}$, Simin Liu, $M D, S c D^{5}$, Braxton D. Mitchell, $P h D^{1}$, and Huichun $X u, M D, P h D^{1}$

Objective To identify stroke susceptibility variants, genes and pathways for ischemic stroke (IS) in young adults. Background The genetic architecture of IS is poorly understood and may differ by age of onset. To identify stroke susceptibility variants, genes and pathways for IS in young adults we genotyped participants in the Genetics of EarlyOnset Stroke (GEOS) with an exome array to interrogate the protein-coding portion of genes. Design/Methods The GEOS Study was initiated as a biracial population-based study of cases with first-ever ischemic stroke 15 to 49 years of age ( $\mathrm{n}$ $=828)$ and controls $(\mathrm{n}=850)$. All participants had prior GWAS and underwent Illumina exome-chip genotyping. Mixed model regression in EMMAX was conducted to calculate variant-specific associations using pooled EuropeanCaucasians (EUR) and African-Americans (AA) samples, with population structure controlled by kinship matrix relationships using prior GWAS data. Gene-based burden testing and meta-analysis were performed using seqMeta with ethnicity-specific GWAS PCs. In both analyses covariates included age and gender. Analyses of all-stroke and as stratified by stroke subtype were performed. This was followed by Ingenuity Pathway Analyses on the top genes. Results While no individual SNPs reached chip-wide significance $\left(3 \times 10^{-7}\right)$, several were near, including one missense variant in TRAPPC11 (rs67383011, $p=1.7 \times 10^{-6}$ ). Gene-based burden testing using a filter (cumulative MAC $>40$, \# of SNPs per gene $\geq 2$, missingness $=0$ ) was performed on 6934 genes in EURs and 9423 genes in AAs. While several interesting genes were identified for all-stroke, none reached statistical significance in the ethnicity-stratified $\left(p=3.3 \times 10^{-4}\right)$ or metaanalyses $\left(p=1.1 \times 10^{-4}\right)$. Gene-based subtype-specific analyses identified NAT10 $\left(p=3.8 \times 10^{-6}\right)$ as associated with small vessel stroke subtype at a genome-wide significance level $\left(p=4.7 \times 10^{-6}\right)$. Pathway analyses on the top 438 genes $(p<0.05)$ from the gene-based results identified several metabolism and signaling pathways, including: (1) nuclear receptor signaling related to lipid/glucose metabolism; (2) neurotransmitter glutamate receptor signaling, and notably; (3) neurodevelopment notch signaling. Efforts in additional data sets to replicate these findings are ongoing. Conclusions Exome-based analyses in the setting of early-onset stroke is a promising methodology to identify novel genetic risk variants, loci and pathways.

Contact Email: jcole@som.umaryland.edu

\section{China Kadoorie Biobank: Opportunities for research into the aetiology of stroke}

\author{
Zhengming Chen, DPhil ${ }^{1,2}$; on behalf of the China Kadoorie \\ Biobank collaborative group
}

Objective To assess genetic and non-genetic determinants of stroke types in Chinese adults. Background Stroke is the leading cause of death and disability in China. Several major modifiable risk factors for stroke are known, but they do not explain the large variations in the age-specific rates of stroke 
between different populations. Moreover, little is known about genetic determinants of stroke types and their main subtypes. Large prospective biobank studies in diverse populations, with heterogeneity in risk exposures, disease rates and genetic architecture, are essential for assessing the causal relevance for stroke (and other major diseases) of lifestyle, environment, biochemical and genetic factors, and their interactions. Design/Methods The China Kadoorie Biobank recruited 512,000 adults from 10 areas across China during 2004-2008, with extensive data collected by questionnaire and physical measurements and with long-term storage of blood samples. All the participants are followed up for fatal and non-fatal stroke and other disease outcomes through electronic linkages to death registries and national health insurance systems. Results By January 1, $2017>40,000$ incident cases of ischaemic stroke and $>8,000$ of intracerebral haemorrhage had been recorded, with $>90 \%$ of diagnosis confirmed by CT/MRI. To further improve the accuracy of stroke diagnosis and sub-phenotyping (e.g., TOAST subtypes of ischaemic stroke), all reported cases of stroke (along with several other diseases) are being adjudicated systematically, involving retrieval of the original hospital records and review by clinical specialists. These exposure and health outcome data are now being complemented by cohort-wide genotyping (100K completed and genotyping of the remaining $412 \mathrm{~K}$ to be completed in 3 years), along with whole genome sequencing and metabolomic/proteomic assays in nested case-control study of stroke types. Many major findings are now emerging, some expected and some intriguingly unexpected but novel, including the role of adiposity, lipids and alcohol in the aetiology of stroke types. Conclusions $\mathrm{CKB}$ will advance understanding of stroke aetiology, reveal novel insights about mechanistic basis of stroke types, facilitate drug target validation and discovery, and improve risk prediction and development and delivery of precision medicine for stroke.

Contact Email: zhengming.chen@ndph.ox.ac.uk

\section{Genome-wide interaction study with sex identifies novel loci for intracerebral hemorrhage risk}

Jaeyoon Chung, $P h D^{1,2}$, Bailey Montgomery, $B S^{1,3}$, Jonathan Rosand, $M D^{1,2,3,4}$, and Christopher D. Anderson, $M D^{1,2,3,4}$

Objective We hypothesize that sex differences in ICH risk may include a genetic component. Understanding these sex-stratified genetic risk contributions could inform on sex-specific disease mechanisms and therapies. Background Intracerebral hemorrhage $(\mathrm{ICH})$ is a complex disease with substantial heritability $\left(\mathrm{R}^{2}=30 \%\right)$. While there is increasing awareness of differences in $\mathrm{ICH}$ risk between men and women, sex-specific determinants of $\mathrm{ICH}$ risk remain unclear. Design/Methods We performed a genome-wide interaction test between genetic variants and sex on ICH risk by location using three ICH datasets totaling 797 lobar and 1,005 non-lobar ICH cases and 1,711 controls. All datasets were imputed to the TOPMed reference panel. Interaction between genetic variants and sex was tested separately by dataset and were then meta-analyzed. Age at onset for cases and age at last exam for controls as well as the principal components for population structure were used as covariates in all analyses. Results We observed two genome-wide significant sex-interacting associations $\left(p<5.0 \times 10^{-8}\right)$, one within SLIT3 (rs2337552; minor allele frequency $[\mathrm{MAF}]=39 \% ; p=1.0 \times$ $10^{-8}$ ) for all ICH, and one upstream of GPR26 (rs908468; MAF $=22 \% ; p=2.1 \times 10^{-8}$ ) for non-lobar ICH only. Rs2337552 showed similar associations in both $\mathrm{ICH}$ subtypes (lobar ICH, $p$ $=1.4 \times 10^{-7}$; non-lobar ICH, $\left.p=2.0 \times 10^{-5}\right)$, while rs908468 showed minimal association with lobar ICH $\left(p=4.4 \times 10^{-3}\right)$. In sex-stratified association tests, both variants showed suggestive associations $\left(p<10^{-5}\right)$ in both males and females, but the effect directions of those SNPs differ between men and women. Conclusions SLIT3 and GPR26 are hormone receptors for estrogen and serotonin, respectively, and serotonin has been previously associated with stroke risk in women via a presumed pro-inflammatory mechanism. SLIT3 promotes vascular network formation and has been associated with sex-specific traits including ovarian and breast cancers and age at menarche. GPR26 has been identified in previous genetic studies of atherosclerosis risk. We have begun to replicate these associations in independent datasets.

Contact Email: rosand@chgr.mgh.harvard.edu

\section{Rare missense variants in intracerebral hemorrhage}

Jaeyoon Chung, $\mathrm{PhD}^{1,2}$, Minsup Kim, $\mathrm{PhD}^{3}$, Jonathan Henry, $B S^{1,4,5}$, Bailey Montgomery, BS $S^{1,4}$, Jonathan Rosand, $M D^{1,2,4,5}$, and Christopher D. Anderson, $M D^{1,2,4,5}$

Objective We hypothesize that novel rare coding variants in COL4A1 and COL4A2 may be responsible for diseaseassociated common variant signals at $13 \mathrm{q} 34$. Background Common genetic variations in COL4A1 and COL4A2 at the 13q34 locus have been previously associated with a broad spectrum of cerebral small vessel diseases, including $\mathrm{ICH}$, white matter hyperintensity, and small vessel ischemic stroke, as well as coronary artery disease. Design/Methods We performed targeted primer-based sequencing across $559 \mathrm{Kbp}$ at $13 \mathrm{q} 34$ including COL4A1 and COL4A2 among 2,133 individuals (1,055 ICH cases and 1,078 controls) and selected rare coding variants with predicted functional impact and appearance in at least two $\mathrm{ICH}$ patients but not controls. Sequence annotation for minor allele frequency (MAF) and functional impact and categories was conducted using gnomAD and the Ensembl Variant Effect Predictor (VEP). We also examined phenomic information for carriers of identified coding variants using the Geno2MP database. We further assessed model protein structures of $\mathrm{CO}$ L4A1 and COL4A2 using protein homology modeling and 
molecular dynamic simulation (across 500 nanoseconds). For comparisons of heterotrimeric triple helix models of COL4A1 and COL4A2, we used the conventional type IV collagen sequence (protein data bank [PDB] ID: 2CUO). Results We identified two missense variants in COL4A1 and COL4A2 appearing in at least two ICH cases but not in controls: (1) rs138269346 (p.I110T, COL4A1) in four subjects (two lobar and two non-lobar ICH patients); (2) rs201716258 (p.H203L, COL4A2) in two subjects (one lobar and one non-lobar ICH patient). Both variants were classified as pathogenic in gnomAD and VEP based on low observed frequency (MAF $<0.00035$ in EUR) and predicted functional impact (probably or possibly damaging). Individuals harboring these two variants in Geno2MP expressed phenotypic abnormalities in the cardiovascular system, eye, nervous system, and musculature. The mutant amino acids encoded by these two variants appear within the triple helix domains. We observed substantial changes in free energies of the two triple helices (free energy change $[\mathrm{kcal} / \mathrm{mol}]$ by the variants; rs138269346: -6.7 and rs201716258: +19.78) compared with wildtype. Conclusions We have identified two rare coding variants in COL4A1 and COL4A2 that appear only in ICH cases but not in controls in our dataset of over 2,000 individuals. Our annotation and simulation studies imply that these variants are highly functional, with amino acids changes that disrupt in the triple helices complexed by COL4A1 and COL4A2. Further replication is needed, and biological experiments will examine the contribution of these variants to type IV collagen function in vitro.

Contact Email: rosand@chgr.mgh.harvard.edu

\section{Obesity increases risk for lobar and deep ICH: a genetic and mendelian randomization analysis}

Sandro Marini, $M D^{1}$, Jordi Merino, $P h D^{1}$, Jaeyoon Chung, $P h D^{1}$, Jose C. Florez, $M D, P h D^{1,2}$, Jonathan Rosand, $M D, M S^{1,2,3}$, and Christopher D. Anderson, $M D, M M S c^{1,2,3}$

Objective We aim to assess whether obesity is causally associated with intracerebral hemorrhage. Background Epidemiological studies on the relationship between intracerebral hemorrhage (ICH) and obesity have presented conflicting results. Human genetics provides an alternative approach to determining whether obesity is likely to represent an unconfounded risk factor for ICH and can also highlight potentially casual molecular pathways leading from obesity to ICH. Design/Methods We used summary statistics from GWAS of ICH (Woo et al. Am J Hum Genet. 2014) and the latest GWAS for body fat distribution measured by body mass index (BMI), waist-to-hip ratio (WHR), and body fat percentage (BFP) (Pulit et al. Hum Mol Genet. 2019 and UK Biobank). For each anthropometric trait, we pruned for linkage disequilibrium and computed a polygenic risk score to assess trait-specific effects on non-lobar and lobar ICH risk.
We also used Mendelian randomization (MR) to explore casual pathways between the anthropometric traits and ICH. We declared statistical significance at $p=0.004$ after Bonferroni correction. Results Genetic predisposition to higher BMI, WHR, and BFP were associated with increased risk of non-lobar ICH (Odd Ratio [OR] for BMI = 2.06, $p=1.20 \times$ $10^{-41}$; OR for WHR $=1.96, p=5.98 \times 10^{-23}$; OR for BFP = $\left.2.04, p=1.35 \times 10^{-22}\right)$, and lobar ICH (OR for BMI $=1.54, p$ $=1.17 \times 10^{-13}$; OR for WHR $=2.13, p=6.26 \times 10^{-24}$; OR for $\left.\mathrm{BFP}=2.19, p=1.33 \times 10^{-22}\right)$. MR analysis demonstrated that higher BMI was independently associated with risk of nonlobar ICH (Egger regression OR $=2.25, p=0.0002$; Weighted Median regression OR $=1.91, p=6.79 \mathrm{e}-07) \cdot p$ for MR-Egger intercept was $1.01 \times 10^{-5}$ for WHR and $8.82 \times 10^{-06}$ for BFP, suggesting that hidden pleiotropy may affect the relationship between these anthropometric traits and $\mathrm{ICH}$. Conclusions Obesity is associated with both non-lobar and lobar ICH. Even if the anthropometric trait that best captures this association is uncertain, our results suggest that increases in BMI may lie within a causal pathway that increases non-lobar ICH risk.

Contact Email: CDANDERSON@PARTNERS.ORG

\section{NOTCH3 exon skipping as}

\section{a rational therapeutic approach for CADASIL: lessons from a family with naturally occurring exon 9 skipping}

Gido Gravesteijn, MD, MSc', Johannes G. Dauwerse ${ }^{2}$, Ingrid Hegeman ${ }^{3}$, Gwendolyn Brouwer, $\mathrm{MSc}^{2}$, Aat A. Mulde ${ }^{4}$, Carolina R. Jost, $\mathrm{PhD}^{4}$, Sjoerd van Duinen ${ }^{3}$, Annemieke Aartsma-Rus, $P h D^{2}$, Julie W. Rutten, $M D, P h D^{1,2}$, and Saskia A.J. Lesnik Oberstein, $M D, P h D^{1}$

Objective To determine the clinical features and vascular pathology in skin biopsy of individuals with naturally occurring NOTCH3 exon skipping. Background CADASIL is a small vessel disease (SVD), caused by cysteine altering missense mutations in the NOTCH3 gene, which result in $\mathrm{NOTCH} 3$ protein aggregation in the (cerebro) vasculature. We recently described a rational therapeutic approach for CADASIL, aimed at counteracting NOTCH3 aggregation via antisense oligonucleotide (AON)-mediated skipping of mutated $\mathrm{NOTCH} 3$ exons. We demonstrated the feasibility of this approach in vitro, but in vivo experiments are so far hampered by inefficient delivery of the AONs. Here, we present a family with naturally occurring exon 9 skipping. Design/Methods All family members had brain MRI and skin punch biopsies were taken and processed for fibroblast culture, immunohistochemistry and electron microscopy. Exon skipping was analysed using RT-PCR and Sanger sequencing analysis on RNA isolated from skin fibroblasts. Results The index (female, 63 years) was referred because of mild cognitive impairment and white matter hyperintensities 
on brain MRI suggestive of SVD. Gene panel testing revealed a novel NOTCH3 mutation in exon 9 (c.1492G>T, p.Gly498Cys). The mutation is located on the splice donor site of exon 9 and therefore predicted to result in exon 9 skipping. Four additional family members with the mutation were identified, all with a relatively mild phenotype without lacunar strokes. RT-PCR and Sanger sequencing analysis on fibroblast RNA showed abundant presence of exon 9 skip product, but also low levels of mutant NOTCH3 from which exon 9 was not skipped. Skin vessels showed only very minimal NOTCH3 aggregation and no GOM. Conclusions These individuals with naturally occurring NOTCH3 exon skipping have a mild late-onset SVD, likely attributable to their low levels of mutant NOTCH3 expression, which is consistent with the strikingly mild NOTCH3 aggregation in their skin vasculature. These findings also suggest that the abundant skipped NOTCH3 does not contribute to the SVD phenotype, supporting the potential feasibility of NOTCH3 exon skipping as a therapeutic strategy in CADASIL.

Contact Email: lesnik@lumc.nl.

\section{Hemostatic genes exhibit a high degree of allele-specific regulation in liver}

Martina Olsson Lindvall, $P h D^{1}$, Lena Hansson, $P h D^{2,3}$, Sofia Klasson, $\mathrm{MSE}^{1}$, Marcela Davila Lopez, $\mathrm{PhD}^{4}$, Christina Jern, $M D, P h D^{1}$, and Tara M. Stanne, $P h D^{1}$

Objective We aimed to identify novel cis-regulatory variants involved in regulating hemostatic genes by analyzing allele-specific expression (ASE) in human liver samples. Background Tight regulation of hemostatic genes in the liver is crucial to avoid thrombus formation which can lead to ischemic stroke. Publically available expression quantitative trait loci (eQTL) datasets currently have low coverage of eQTLs in hemostatic genes in human liver tissue. ASE analysis is an alternative method to quantify cis-regulatory variation, applicable even in a limited sample size. ASE has an advantage over eQTL analyses as it relies on comparisons within an individual, therefore sources of error between individuals are eliminated. To the best of our knowledge, no studies have analyzed ASE in hemostatic genes in human liver. Design/Methods Biopsies of human liver tissue and blood were collected from adults undergoing liver surgery at the Sahlgrenska University Hospital ( $\mathrm{n}$ = 17). Genomic DNA (gDNA) and total RNA were isolated. A targeted approach was used to enrich and sequence 35 hemostatic genes for single nucleotide polymorphisms (SNP) analysis (gDNAseq) and construct individualized genomes for transcript alignment. The allelic ratio of transcripts from targeted RNAseq was determined via ASE analysis. Public eQTL and GWAS data were used to assess novelty and importance of the ASE SNPs for relevant traits/diseases. Results Sixty percent of the genes studied showed allelic imbalance across 53 SNPs. Of these, 7 SNPs were previously validated in liver eQTL studies. For 32 with eQTLs in other cell/tissue types, this is the first time genotype-specific expression is demonstrated in liver, and for 14 ASE SNPs, this is the first ever reported genotype-expression association. A total of 29 ASE SNPs were previously associated with the respective plasma protein levels and 16 ASE SNPs to other relevant GWAS traits including stroke. Conclusions Our study provides a comprehensive ASE analysis of hemostatic genes and insights into the regulation of hemostatic genes in human liver. In the future, we envisage that this data set could be used by the hemostatic research community.

Contact Email: tara.stanne@gu.se

\section{Genetics of white matter hyperintensity stratified by hypertension status: The CHARGE Consortium}

Y. Saba, $M \mathrm{Sc}^{1,2,3}$, E. Hofer, $P h D^{4,5}$, M. Sargurupremraj, $P h D^{2,3}$, H. Adams, $P h D^{6}$, L. J. Launer, $\mathrm{PhD}^{7}$, A. Ikram, $\mathrm{MD}^{6}$, P. Nyquist, $M D, P h D^{8}$, M. Fornage, $P h D^{9}$, S. Seshadri, $M D^{10,11}$, S. Debette, $M D, P h D^{2,3,10,12}$, and H. Schmidt, $M D^{1}$; on behalf of the neuroCHARGE Working Group

Objective We seek to identify novel risk variants for WMH burden, by performing a genome-wide association study (GWAS) of WMH burden stratified by hypertension status in the general population. Background White matter hyperintensities (WMH) represent the most common MRImarkers of cerebral small vessel disease. They are associated with increased risk of stroke, dementia, and death. Besides age, hypertension is the major risk factor for $\mathrm{WMH}$ and heritability has been estimated up to $70 \%$. Design/Methods In total we included 15,595 middle-aged to older participants of European descent from 17 population-based cohorts participating in the CHARGE consortium, comprising 5,695 normotensives (NT) and 9,900 hypertensives (HT). Within the HT group a subgroup of severe hypertensives (SHT) with SBP $\geq 160 \mathrm{mmHg}$ and DBP $\geq 100 \mathrm{mmHg}(\mathrm{N}=3,362)$ was defined. WMH was quantified on MRI by automated segmentation methods except in two studies where a semiquantitative a visual grading scale was used. We estimated WMH heritability both in a familial and population-based setting using SOLAR and GCTA. GWAS adjusted for age, sex, intracranial volume, and principal components of population stratification were performed in each cohort, followed by meta-analyses within each blood pressure stratum. SNPs reaching $p<5 \times 10^{-8}$ were considered genome-wide significant. Results Heritability estimates of WMH appeared higher in NT than HT, both in related individuals (NT: h2 $=0.61, p=$ $1.78 \times 10^{-5}$; HT: h2 $\left.=0.43, p=1.25 \times 10^{-2}\right)$ and in unrelated individuals (NT: h2 $=0.68, p=7 \times 10^{-3}$; HT: h2 $=0.43, p=2 \times$ 
$10^{-2}$ ). The GWAS identified 3 genome-wide significant loci for WMH in HT, including two novel, on chr5q35.1 (FOXI1, $p=$ $5.23 \times 10^{-9}$ ) and chr14q32.2 (EVL, $p=4.26 \times 10^{-8}$ ), and a previously known locus on chr17q25.1 (TRIM65, $p=5.82 \times$ $\left.10^{-17}\right)$. The effect size of these risk variants corresponds to an age effect of up to 1.2 years. In NT and SHT no signal reached genome-wide significance. A meta-regression showed significant association with age in HT for the 3 genome-wide significant loci and with SBP for the chr5q35.1 and chr17q25.1 loci. Conclusions This first WMH GWAS stratified by hypertension status in the general population led to the identification of novel risk loci, the replication of which is underway. In addition, among hypertensives, our results indicate a modifying effect of increasing age and SBP (figure 1).

Contact Email: stephanie.debette@u-bordeaux.fr

\section{Whole exome sequencing analysis of 22 young stroke patients with familial clustering of stroke}

A. Ilinca, $M D^{1,2}, N$. Martinez-Majander, $M D, M S c^{3}, S$. Samuelsson, $M \mathrm{Sc}^{4}$, P. Piccinelli, $M D, P h D^{4}$, K. Truvé, $P h D^{5}$, J.W. Cole, $M D, M S^{6,7}$, S.J. Kittner, $M D, M P H^{6.7}$, M. Soller, $M D$, $P h D^{8}$, U. Kristoffersson, $M D, P h D^{4}$, T. Tatlisumak, $M D, P h D^{9}$, A. Puschmann, $M D, P h D^{1,2}$, J. Putaala, $M D^{3}$, and A. Lindgren, $M D, P h D^{1,2}$
Objective We intend to investigate how to appropriately detect Mendelian associated stroke. Background Apart from few rare monogenic disorders, exact genetic mechanisms related to stroke risk are mostly unknown. Due to demonstrated stronger heritability, monogenic factors may be of special interest in younger stroke patients. New methods for genetic analyses are rapidly evolving, allowing a more systematic investigation of human exome. Design/Methods Twenty-two probands under 56 years at their first stroke episode from multi-incident families underwent whole exome sequencing (WES). Only exonic or splice-site variants with a minimum allele frequency $<1 \%$ were considered. Data interpretation used a comprehensive panel of genes associated with stroke and the related stroke subtype. We did co-segregation analysis to evaluate suspected pathogenicity of variants of unknown significance in known stroke-genes. In two pedigrees with embolic stroke of undetermined source (ESUS), 16 additional relatives were also examined by WES. Additional affected and unaffected family members were genotyped in 5 families. Results Seven probands carried possibly pathogenic mutations in known genes associated with their stroke subtype. COL4A2 c.3368A $>$ G (p.Glu1123Gly) was detected in two probands but did not co-segregate with small vessel disease in the families. A novel mutation in the highly conserved JAK2kinase insertion loop domain JAK2 c.3188G $>$ A (p.Arg1063His) segregated with ESUS and pro-thrombotic status. The two ESUS pedigrees that were further genotyped showing possible pathogenic variants in genes not previously associated with stroke: EFHB c.990_991delAGinsGA

Figure 1 (Abstract 11) WMH GWAS stratified by hypertension; among hypertensives results indicate a modifying effect of increasing age and SBP

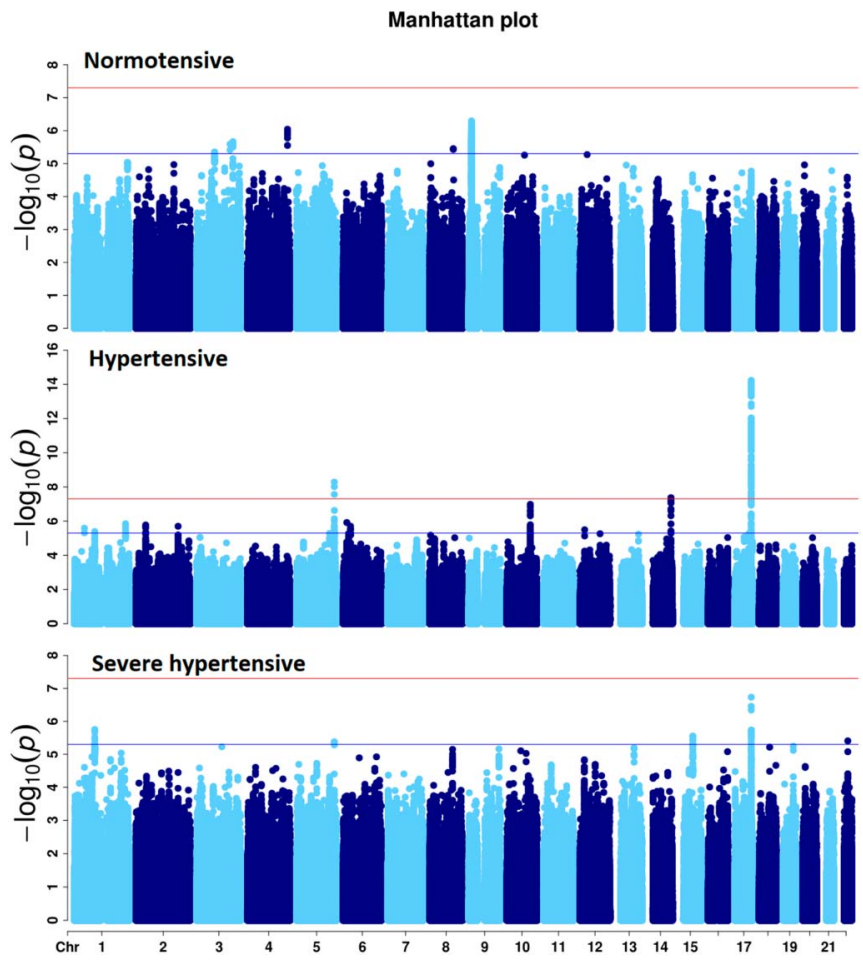


(p.Val331Ile), HYDIN c.10441C $>$ T (p.Arg3481Trp), GPR142 c.148C $>$ G (p.Leu50Val) in the first family; and LRRC1 c.808A> G (p.Ile270Val), PI4K c.3712C >A p.(Leu1238Met) in the second family. Conclusions Our results suggest there may be additional, yet unidentified genetic causes of monogenic stroke. Alternatively, familial clustering of stroke may result from oligogenic mechanisms.

Contact Email: Arne.G.Lindgren@skane.se

\section{The genetic architecture of MRI-derived extremes of cerebral} small vessel disease

Aniket Mishra, $P h D^{1}$, Dina Vojinovic, $M D, P h D^{2}$, Hideaki Suzuki, $M D^{3}$, Josh Bis, $P h D^{4}$, Nuno R. Zilhao, $P h D^{5}$, Xueqiu Jian, $P h D^{6,7}$, Qiong Yang, $P h D^{8,9}$, Wei Zhao, $P h D^{10}$, Sarah Harris, $P h D^{11}$, Yasaman Saba, MSc ${ }^{12}$, Shuo Li, $P h D^{8}$, Murali Sargurupremraj, PhD ${ }^{1}$, Traci M. Bartz, MSc ${ }^{4}$, Edith Hofer, $P h D^{13,14}$, Ganesh Chauhan, $P h D^{1,15}$, Fen Peng, $P h D^{6}$, Thomas H. Mosley, PhD ${ }^{16,17}$, Bruce M. Psaty, MD, PhD ${ }^{18,19,20,21}$, Lenore J. Launer, $P h D^{22}$, Eric Boerwinkle, $P h D^{6}$, Yicheng $Z h u, M D^{23}$, Bernard Mazoyer, $\mathrm{MD}, \mathrm{PhD}^{24}$, Mark Lathrop, $\mathrm{PhD}^{25}$, Anne Joutel, $M D, P h D^{26}$, Christophe Tzourio, $M D, P h D^{1,27}$, Jennifer $A$. Smith, PhD, $M P H^{28}$, Michelle Luciano, $P h D^{11}$, Reinhold Schmidt, $M D^{13,14}$, M. Arfan Ikram, $M D^{2,29}$, Helena Schmidt, $M D^{11}$, Vilmundur Gudnason, $M D, P h D^{5}$, Paul Matthews, $M D$, $D P h i l^{3}, W$. T. Longstreth Jr., $M D^{30}$, Myriam Fornage, $P h D^{5}$, Cornelia Van Duijn, $P h D^{2,31}$, Sudha Seshadri, $M D^{7,32}$, and Stephanie Debette, $\mathrm{MD}, \mathrm{PhD}^{1,32,33}$

Objective To perform a gene-mapping study of MRI-markers of cerebral small vessel disease (SVD) using a composite extreme phenotype design combining extreme distributions of white matter hyperintensity (WMH) burden and presence or absence of lacunes (extreme-SVD). Background WMH and lacunes are heritable and common in older community persons. Genome-wide association studies (GWAS) reported common variants in five loci to be associated with WMH burden in the general population and in four additional loci in stroke patients. No genome-wide significant loci were reported in association with lacunes. Design/Methods Overall 17 population-based cohorts of middle-aged to older persons with available MRI measurements and genome-wide genotyping $(\mathrm{N}=19,056)$, whole exome sequencing (WES, $\mathrm{N}$ $=5,098)$ or exome chip $(\mathrm{EC}, \mathrm{N}=12,666)$ data contributed to this study. We performed meta-analyses of GWAS, genebased tests (using VEGAS2) and multi-trait association studies (jointly with WMH burden, MRI-defined brain infarcts and stroke using MTAG) for common variant genemapping of extreme-SVD. We also used WES/EC data to test association of single variants and burden of rare and low frequency variants with extreme-SVD. We performed partitioned heritability analyses to identify enriched brain cells and regions and SMR/HEIDI tests to identify pleiotropic association of gene expression in brain with risk of extremeSVD. Results We identified 12 genome-wide significant associations at $1 \mathrm{q} 21.2,2 \mathrm{p} 16.1,2 \mathrm{p} 21,2 \mathrm{q} 32.1,2 \mathrm{q} 33.2,6 \mathrm{q} 25.1$, 7q36.1, 10q24.33, 12q24.11, 14q32.2, 16q24.1, and 17q25.1. The VEGAS2 gene-based test identified gene-wide significant association at one additional locus, 17q21.3. The gene-based burden test of rare and low frequency variants identified PHACTR4 $\left(p=1.96 \times 10^{-5}\right)$ as the top gene. The candidate study of five familial SVD genes identified significant association of common variants in COL4A1/2 (rs9515201, $p=9.43$ $\times 10^{-4}$ ) and HTRA1 (rs2293871, $\left.p=2.22 \times 10^{-5}\right)$, and burden of rare and low frequency variants in NOTCH3 $(p=$ 0.017 ) with extreme-SVD. The partitioned heritability analyses showed extreme-SVD heritability enrichment in highly expressed genes in astrocytes and in caudate nucleus. The SMR/HEIDI tests showed pleiotropic association of extremeSVD common risk variants at the 17q25.1 locus with TRIM47, RP11-552F3.10 and WBP2 expression in brain. Conclusions We report a multi-cohort gene-mapping study on extreme-SVD that expands our understanding of the genetic risk architecture of cerebral SVD.

Contact Email: stephanie.debette@u-bordeaux.fr

\section{An analysis of polygenic risk score for T2D and LDL-C in posterior vs anterior circulation ischemic stroke}

P. Frid, $M D^{1}$, A.K. Giese, $M D^{2,3}$, J. Wasselius, $M D, P h D^{4}, M . D$. Schirmer, $P h D^{2,5,6}$, K.L. Donahue, $B S^{2}, L$. Cloonan, $B A^{2}$, R. Irie, $P h D^{7}$, S. Winzeck, $M S^{7}$, M.J.R.J. Bouts, $P h D^{7}$, E.C. McIntosh, $M A^{7}$, S. J. Mocking ${ }^{7}$, A.V. Dalca, $P h D^{5,7}$, R. Sridharan, $P h D^{5}$, H. Xu, $M D, P h D^{8}$, E. Giralt-Steinhauer, $M D, P h D^{10}$, L. Holmegaard, $M D^{9}$, K. Jood, $M D, P h D^{9}$, J. Roquer, $M D, P h D^{10}$, J.W. Cole, $M D^{11}$, P.F. McArdle, $P h D^{8}$, J.P. Broderick, $M D^{12}$, J. Jimenez-Conde, $M D, P h D^{10}$, C. Jern, $M D, P h D^{9}$, B.M. Kissela, $M D, M S^{12}$, D.O. Kleindorfer, $M D^{12}$, R. Lemmens, $M D, P h D^{13}$, J. F. Meschia, $M D^{14}$, T. Rundek, $M D, P h D^{15}$, R.L. Sacco $M D$, $M S^{15}$, R. Schmidt, $M D^{16}$, P. Sharma, $M D, P h D^{17}$, A. Slowik, $M D, P h D^{18}$, V. Thijs, $M D, P h D^{19}$, D. Woo, $M D, M S^{12}$, B. B. Worrall, $M D, M S c^{20}$, S.J. Kittner, $M D, M P H^{11}$, B.D. Mitchell, $P h D, M P H^{8,21}$, J. Petersson, $M D, P h D^{1}$; J. Rosand, $M D$, $M S^{2,3,7,22,23}, P$. Golland, $P h D^{5}, O . W u, P h D^{7}$, N.S. Rost, $M D$, $M P H^{2}$, and A. Lindgren, $\mathrm{MD}, \mathrm{PhD}^{24}$; on behalf of the Stroke Genetics Network (SiGN) and the MRI-Genetics Interface Exploration (MRI-GENIE) Study

Objective To explore the hypothesis that ischemic stroke risk factors contribute differentially to strokes occurring in posterior circulation ischemic stroke (PCiS) versus anterior circulation ischemic stroke (ACiS). Background Endothelial function and signaling differ between the vascular beds of the anterior and posterior circulation, raising the possibility that conventional stroke risk factors may contribute to strokes occurring in these vascular beds. Indeed, recent studies 
support an elevated risk of PCiS in diabetic patients. In this study, we use polygenic risk scores (PRS) to test if genetic risk for stroke risk factors differs between PCiS and ACiS. Design/Methods Stroke phenotyping was performed in 3,300 stroke cases from the MRI-GENIE cohort. These were classified as PCiS or ACiS based on the location of acute ischemic lesions on DWI. Exclusion criteria were lesions in both vascular territories, no DWI lesions, difficulty in classifying vascular territory, and imaging quality issues. To refine the stroke phenotypes further, cases with supratentorial posterior lesions with an ipsilateral F-PCA were excluded, as were all cases with atrial fibrillation. We identified 450 PCiS and 1,036 ACiS cases for whom genome-wide genotyping was available through SiGN. Publicly available GWAS results were used to generate PRS. Mean PRS were compared between PCiS and $\mathrm{ACiS}$ and between each of these phenotypes and non-stroke controls using logistic regression, adjusting for sex and 10 principal components capturing population ancestry. Results PRS for genetic risk of type 2 diabetes (T2D) was significantly associated with both PCiS and ACiS compared to non-stroke controls $(p=0.0048$ for PCiS vs control, $p=0.0007$ for ACiS vs control) but did not differ significantly between PCiS and ACiS $(p=0.83)$. Additionally, we found no evidence for a differential effect of an LDL-C PRS score for ACiS compared to PCiS $(p>0.05)$. Conclusions PRS analyses did not show a differential effect of T2D or LDL-cholesterol on ACiS vs. PCiS. Analyses are ongoing of PRS for other risk factors, including obesity, hypertension, endothelial dysfunction, and inflammation.

Contact Email: Arne.G.Lindgren@skane.se

\section{Regions of shared genetic structure between DWMH, PVWMH, and stroke phenotypes}

Nicola J. Armstrong $P h D^{1}$, Karen A. Mather $P h D^{2}$, Stephanie Debette, $M D^{3}$, Muralidharan Sargurupremraj, $P D^{3}$, and Paul Nyquist, $\mathrm{MD}, \mathrm{MPH}^{4}$; for CHARGE, ENIGMA, MEGASTROKE, and ISGC

Objective To complete a genetic correlation analysis, to identify genes and regions of genetic overlap, shared between ischemic stroke, ICH, and PVWMH, DWMH through comparison of summary data from two large GWAs. Background Two sub-classifications of white matter hyperintensities, deep (DWMH) and periventricular (PVWMH), are thought to reflect both common and different pathophysiology and genetic underpinnings. DWMH and PVWMH may share genetic risk with stroke. We assessed these relationships using GWAS summary data from MEGASTROKE $(n=520,000)$ and our own DWMH and PVWMH study ( $\mathrm{n}=24,517)$. Design/Methods LDSC was used to assess genetic correlations between DWMH, PVWMH and stroke phenotypes including all stroke (AS), ischemic stroke (IS), cardio embolic
(CE), large artery stenosis (LAS), small vessel disease (SVD), deep ICH (deep), and lobar ICH (lobar). Regional level shared influence was investigated using the GWAS-pw (Pickrell et al.) method between stroke and $\mathrm{WMH}$ subtypes, as well as HESS (Shi et al) for directionality. Results Significant genetic correlations were observed for DWMH and PVWMH $[0.9274(p=1.1 \mathrm{e}-65)]$, DWMH and AS $[0.3864(p=0.0001)]$, PVWMH and AS $[0.3803(p=$ 9.69e-07)], IS and DWMH [0.2911 $(p=0.004)]$, IS and PVWMH $[0.3186(p=3.47 \mathrm{e}-05)]$, SVD and DWMH $[0.515$ $(p=0.0137)]$, SVD and PVWMH $[0.7088(p<5.21 \mathrm{e}-05)]$, Deep ICH and DWMH $[0.611(p=0.0055)]$, Deep ICH and PVWMH $[0.4041(p=0.0145)]$. No significant correlation between either WMH phenotype and CE, LAS, and lobar were observed. Using GWAS-pw, we identified significant regions of shared genetic influence between: (1) both DWMH and PVWMH and AS and IS (chr7) (2) PVWMH and CE (chr7); (3) PVWMH and SVD (chr13; chr2); (4) DWMH and SVD (chr6); (5) PVWMH and deep ICH (chr 13; chr 20). There were no significant regions between either phenotype and lobar ICH. Conclusions We observed significant genetic overlap between PVWMH and DWMH and all stroke subtypes. Of particular interest is the strong association of PVWMH with SVD and deep ICH with regions containing putative genes located on chr13 (COL4A1/ COL4A2), chr2 (NBEAL1) and chr 20, (CHD6). Our data suggest shared genetic susceptibilities exist between the periventricular region and deep ICH, SVD and ischemic stroke.

Contact Email: pnyquis1@jhmi.edu

\section{The association of arterial stiffness with hypertension and stroke: a Mendelian randomization study}

Kwang-Yeol Park, $M D, P h D^{1}$, Joo-yeon Lee, $M P H, P h D^{2}$, Soo Ji Lee, $M P H, P h D^{2}$, and Joohon Sung, $M D, P h D^{3}$

Objective We aim to investigate the causal association of arterial stiffness with hypertensive brain injury using Mendelian randomization. Background Previous studies have reported that arterial stiffness is associated with hypertension and cerebral small vessel disease mainly through crosssectional study. Design/Methods We performed a Mendelian Randomization (MR) analysis using the GWAS results data from UK BioBank (118K individuals for arterial stiffness index [ASI] and $340 \mathrm{~K}$ for $\mathrm{BP}$ variables) for exposure variables and SiGN consortium data (17K for ischemic stroke and $32 \mathrm{~K}$ as controls). We analyzed the associations of ASI and blood pressure (systolic and diastotic blood pressure: SBP and DBP, respectively) with three subtypes of ischemic stroke according to TOAST classification. We combined two GWAS results with meta-analysis using METAL including genome-wide significant SNPs in MR analysis ( $p$-value $<10^{-5}$ for ASI). For 
MR analyses, "TwoSampleMR" package in $\mathrm{R}$ was used. Results On MR analysis, $1 \mathrm{~m} / \mathrm{s}$ increase in genetically predicted ASI was associated with mean increase of SBP by 0.081 (95\% CI, 0.003-0.16; $p=0.04$ ) and DBP by 0.072 (95\% CI, $0.027-0.12 ; p=0.002)$. Genetically predicted SBP increase by $1 \mathrm{~mm} \mathrm{Hg}$ was associated with small artery occlusion (odds ratio 1.03 ; $95 \% \mathrm{CI}, 1.02-1.05 ; p<0.001$ ), large artery atherosclerosis (odds ratio 1.06; 95\% CI, 1.04-1.08; $p<0.001$ ), and cardiac embolism (odds ratio 1.02; 95\% CI, 1.001-1.03; $p$ $=0.04)$. Also, DBP increase by $1 \mathrm{~mm} \mathrm{Hg}$ was associated with small artery occlusion (odds ratio 1.08; 95\% CI, 1.05-1.11; $p$ $<0.001$ ), large artery atherosclerosis (odds ratio 1.05; $95 \% \mathrm{CI}$, 1.02-1.09; $p=0.001$ ), and cardiac embolism (odds ratio 1.02; 95\% CI, 0.9991-1.05; $p=0.059$ ). Conclusions In conclusion, arterial stiffness is linked to hypertension and ischemic stroke. It should be tested in future trials whether arterial stiffness can be the therapeutic target for stroke.

Contact Email: kwangyeol.park@gmail.com

\section{Genome-wide methylation in the acute phase ischemic stroke}

C. Soriano-Tárraga, $P h D^{1}$, E. Giralt-Steinhauer, $M D, P h D^{1}$, U. Lazcano, $\mathrm{MSc}^{1}$, C. Avellaneda-Gómez, $\mathrm{MD}^{1}$, R.M. VivancoHidalgo, $\mathrm{MD}, \mathrm{PhD}^{1}$, A. Ois, $\mathrm{MD}, \mathrm{PhD}^{1}$, A. Rodríguez-Campello, $M D, P h D^{1}$, E. Cuadrado-Godia, $M D, P h D^{2}, R$. Elosua, $P h D^{2}$, I. Fernández-Cadenas, $\mathrm{PhD}^{3}, \mathrm{~N}$. Cullell, $\mathrm{MSc}^{3}, \mathrm{M}$. Esteller, $\mathrm{MD}$, $\mathrm{PhD}^{4}$, J. Jiménez-Conde, $\mathrm{MD}, \mathrm{PhD}^{1}$, and J. Roquer, $\mathrm{MD}, \mathrm{PhD}^{1}$

Objective To analyze which genes are differentially methylated in the acute phase of ischemic stroke versus controls. These changes could have an important role to orchestrate cellular reactions and gene expression in the acute phase of ischemic stroke. Background DNA methylation (DNAm) is an epigenetic mechanism regulating higher-order DNA structure and gene expression. It is a heritable, but also a reversible addition of a methyl group to the 5-carbon position of cytosine in a cytosine-phosphate-guanine ( $\mathrm{CpG}$ ) context, associated with gene silencing. DNAm is dynamic, varies throughout the life course and its levels are influenced by lifestyle and environmental factors, as well as by genetic variation. Stroke's epigenetics has revealed that stroke patients are globally hypomethylated, epigenetically older than healthy controls and this accelerated aging is strongly associated with ischemic stroke (IS) outcome at 3 months, independently of healthiness, lifestyle, and genetic features. Given its dynamic nature, DNAm could have an important role to orchestrate cellular reactions and gene expression in the acute phase of IS. Design/Methods The discovery cohort included 628 patients in the acute phase of IS, assessed at Hospital del Mar (Barcelona, Spain). We performed a genome-wide methylation study using wholeblood DNA and a population cohort as controls $(\mathrm{N}=346)$. We used Human Methylation 450 and Infinium Methylation EPIC Beadchip arrays (Illumina) to measure DNA methylation. All statistical analyses were adjusted for sex, age, smoking habit, cell count, hyperlipidemia, hypertension, diabetes mellitus, ischemic cardiomyopathy and atrial fibrillation. Results A total of $67 \mathrm{CpGs}$ were significant and differentially methylated in the acute phase of IS versus controls, and successfully replicated in an independent IS cohort $(\mathrm{N}=292)$ using the same arrays. Conclusions These results presented orally in the ISGC workshop meeting.

Contact Email: jjimenez@imim.es

\section{Cerebral phenotypes associated with mutations in Mendelian cerebral small vessel disease genes: A systematic review}

David E. Henshall, $\mathrm{PhD}, \mathrm{MBChB}{ }^{1}$, Mike Chong, $\mathrm{MSc}^{2}$, Nickrooz Grami, $B H S c^{2}$, Isaac Kuan, $P h D^{1}$, Guillaume Paré, $M D$, $\mathrm{MSc}^{2}$, Tim Wilkinson, $\mathrm{MD}, \mathrm{MSc}^{3}$, Blair Wilson, $M D^{1}$, Kirsty Wilson, $\mathrm{PhD}^{3}$, Cathie Sudlow, DPhil, $\mathrm{MSc}^{3}$, and Kristiina Rannikmäe, $M D^{3}$

Objective To identify and quantify cerebral phenotypes associated with Mendelian cerebral small vessel diseases (CSVD) in order to improve our understanding of these disorders. Background CSVD is a major cause of morbidity in the developed world. The majority of cases are sporadic but Mendelian forms account for a significant minority and may offer insights into the mechanisms of sporadic disease. Design/Methods: We: (1) systematically sought publications of cases with mutations in COL4A1/2 (heterozygous, HetZ), TREX1 (HetZ), HTRA1 (HetZ or homozygous, HZ), PITX2 (HetZ), FOXC1 (HetZ), CTSA (HetZ), CECR1 $(\mathrm{HZ}) ;(2)$ extracted number of unique mutations, families and cases, their mean age and sex; (3) quantified cerebral phenotypes for each gene. Results We included 200 publications from 5165 publications screened (figure 2). Mean age ranged from 14 (CECR1) to 61 years (HTRA1 HetZ), and proportion of females from $30 \%$ (HTRA1 HetZ) to $87 \%$ (CTSA). CSVD neuroimaging markers were present in all HTRA1 HZ, PITX2, FOXC1, CTSA cases, and in $92 \%$ HTRA1 HetZ, 49\% TREX1, 40\% COL4A1, 16\% COL4A2, $16 \%$ CECR 1 cases. Stroke/TIA was reported in 58\% HTRA1 HetZ, 43\% CTSA, 33\% CECR1, 26\% COL4A1, 26\% HTRA1 HZ, 20\% FOXC1, 16\% COL4A2, 2\% TREX1 cases. Of stroke cases: $\leq 50 \%$ were lacunar or deep; $\geq 60 \%$ COL $4 A 1 / 2$ stroke cases were haemorrhagic, but this proportion was $\leq 25 \%$ for other genes. Proportion of cases with cognitive impairment ranged from $\leq 10 \%$ (FOXC1, PITX2, CECR1) to $71 \%$ (HTRA1 HZ), with psychiatric problems from $\leq 10 \%$ (COL4A1/2, FOXC1, PITX2, CECR1) to 57\% (CTSA), and with migraine from $\leq 1 \%$ (PITX2, FOXC1, CECR1) to $36 \%$ (CTSA, TREX1). Conclusions While these estimates are heavily influenced by case selection for genotyping, 
Figure 1.

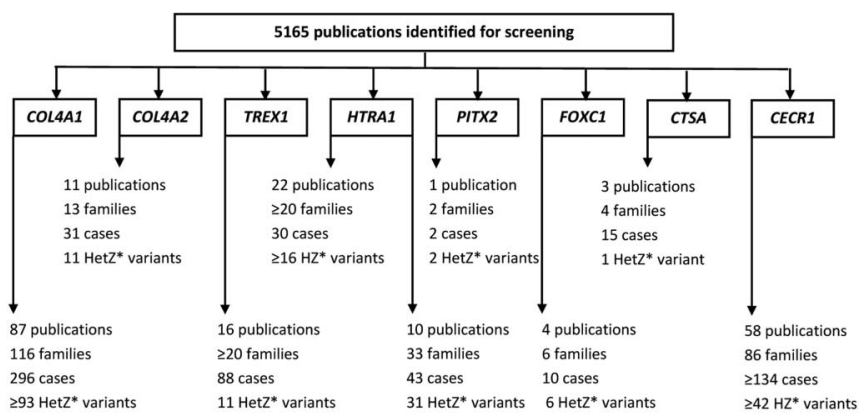

${ }^{*} \mathrm{HZ}$ : homozygous or compound heterozygous; Hetz: heterozygous

publication bias and varying reporting quality, our findings suggest a variable presence and subtype of CSVD phenotype associated with mutations in different genes. Understanding the reasons for these differences may reveal important mechanistic insights into CSVD.

Contact Email: Cathie.Sudlow@ed.ac.uk

\section{The role of haematological traits in stroke and its subtypes}

Eric L. Harshfield, $P h D^{1}$, Matthew C. Sims, $P h D^{2,3}$, Matthew Traylor, $P h D^{1}$, William J. Astle, $P h D^{4,5}$, Willem H. Ouwehand, FMedSci, ${ }^{2,5,6,7,8}$, and Hugh S. Markus, $D M^{1}$

Objective To ascertain causal relationships between a wide range of haematological traits and ischaemic stroke and its subtypes. Background Thrombosis and platelet activation play a central role in stroke pathogenesis, and antiplatelet and anticoagulant therapies are central to stroke prevention. However, whether haematological traits contribute equally to all ischaemic stroke subtypes is uncertain. Furthermore, identification of associations with new traits may offer novel treatment opportunities. Design/Methods We obtained summary statistics from 25 published genome-wide association studies of haematological traits involving over 350,000 individuals, and genetic associations with stroke from MEGASTROKE ( $\mathrm{N}=$ 520,000). Using two-sample Mendelian randomisation we analysed the association of genetically elevated levels of 36 blood cell traits and 48 haemostasis traits with risk of ischaemic (IS), cardioembolic (CES), large-artery (LAS), and smallvessel stroke (SVS). Results Several factors on the intrinsic clotting pathway were significantly associated $(p<1.111 \times$ $10^{-4}$ ) with CES and LAS, but not with SVS (e.g., reduced FVIII activity with IS/CES/LAS; raised FVIII antigen with IS/CES; and increased FXI activity with IS/CES). Elevated plateletcrit was associated with IS and CES. Forty-two further haematological traits were also associated with ischaemic stroke and/or subtypes but did not reach statistical significance. Conclusions These results implicate the intrinsic pathway in pathogenesis of CES and possibly LAS, but not SAS. Plateletcrit may also have a direct causal effect on ischaemic stroke risk. The lack of association with SAS suggests thrombosis may be less important for this stroke subtype. Further investigation of these traits may lead to better understanding of the role of thrombosis in specific stroke subtypes and the identification of targets for novel preventative drugs.

Contact Email: hsm32@cam.ac.uk

\section{The BRAINS study: First analysis of socioeconomic and demographic influence on stroke onset data in Indians and Qataris using the bio- repository of DNA in stroke biobank}

Dhanya Kumar, BSc ${ }^{1}$, Ankita Maheshwari, MSc', Taylor Aurelius, $B S c^{1}$, Thang Han, $M D, P h D^{1}$, Sapna Sharma, $M D^{1}$, Hasan Al Hail, MD, FAAN ${ }^{3}$, P.N. Sylaja, MD, MBBS ${ }^{4}$, Fahmi Khan, MBBS, CABMs ${ }^{3}$, Kameshwar Prasad, $M D, M M S c^{2}$, and Pankaj Sharma, $\mathrm{MD}, \mathrm{PhD}^{1,5}$; on behalf of the BRAINS Collaborators

Objective Determine whether there are socioeconomic and demographic discrepancies of stroke onset in India and the Middle East compared to existing data. Background Genetic information on stroke has been mainly derived from Caucasian cohorts, with little investigation in other ethnic groups, such as Indians and Arabs, who represent $\sim 15 \%$ of the world's population. Moreover, the incidence of stroke in India has doubled while declined by half in Western Europe within the same time period. To increase representation, we established the Bio-Repository of DNA in Stroke (BRAINS), the largest of its kind in the world, to recruit stroke patients of 
Indian ancestry from India, the Middle East (Qatar) and the UK. In preparation of genetic analyses, we present the first analysis of the BRAINS data focusing on impact of socioeconomic influences prior to a genome-wide search. Design/Methods Stroke patients of Indian and Qatari ancestry were recruited at medical sites in India (AIIMS and SCTIMST) and Qatar (HMC). Detailed demographic and socioeconomic data were recorded and blood DNA isolated. Age of stroke onset was compared with patients of European ancestry using ANOVA and between sexes by independent t-test from SPSS $v 23$. Results Data on 3,317 Indian stroke patients (males: 2,238) who suffered ischemic $(n=1,966)$, hemorrhagic ( $\mathrm{n}=982)$, and other subtypes are presented. The mean age of stroke onset in South Asians was significantly lower compared to (Wasay et al., 2014) patients of European ancestry (BRAINS: $62.4 \pm 6.1$ years old, USA: 68 y/o, Italy: 71 y/o; $p=0.046$; Qatari $50.0 \pm 10.5)$. Males were affected at a younger age $(54.3 \pm 7.8 \mathrm{y} / \mathrm{o})$ compared to women $(64.1 \pm 4.7$ $\mathrm{y} / \mathrm{o} ; \mathrm{p}=0.033)$. History of hypertension was the most common risk factor affecting this population $(77.8 \%)$ followed by diabetes (58.7\%) and tobacco usage (45.5\%). Using regression analysis, of the 25 collected socioeconomic indicators, ownership of a car $(n=1,530)$, computer $(n=1,572)$, refrigerator $(n=2,260)$ and mobile device $(n=2,720)$ were distinctive factors allowing division of subjects into quartile classes for comparison by wealth and social class. DNA is now being subjected to GWAS. Conclusions Socioeconomic factors play an important part of stroke onset in those of non-European ancestry. Our repository provides a unique opportunity to undertake a GWAS to identify stroke susceptibility loci in underinvestigated ethnic populations.

Contact Email: Pankaj.Sharma@rhul.ac.uk

\section{Genetically determined serum urate and risk of neurovascular disease}

A. Efstathiadou, $M D, M S c^{1}$, D. Gill, $P h D^{1}$, F. McGrane, MBChB, $M R C P^{2}$, T. Quinn, $M D, B S c^{2}$, and J. Dawson, $M D, B S c^{2}$

Background and Aims Serum urate has been implicated in neurological and vascular disease. However, associations from observational studies may be attributed to confounding factors and reverse causation. The aim of this study was to use Mendelian randomization in targeted investigation of a causal effect of urate on neurological and vascular outcomes. Design/Methods We performed conventional and sensitivity Mendelian randomization analyses to investigate the effect of serum urate on cognitive performance, $\mathrm{Alz}$ heimer's disease, coronary heart disease, myocardial infarction, systolic blood pressure, and ischemic stroke and its main etiological subtypes. Results After exclusion of potentially invalid genetic instruments, higher serum urate was associated with lower cognitive performance (odds ratio per $1 \mathrm{mg} / \mathrm{dl}$ increase in serum urate $0.98 ; 95 \%$ confidence interval 0.96-0.99; $p$-value $=0.02)$ and higher systolic blood pressure (odds ratio $1.03 ; 95 \%$ confidence interval $1.01-1.05 ; p$-value $=0.01)$, with all Mendelian randomization methods having consistent estimates. The previously observed genetic association between higher urate and increased coronary heart disease and myocardial infarction risk was also corroborated. There was no apparent Mendelian randomization association between urate with Alzheimer's disease and any type of ischemic stroke. Conclusions This study provides genetic evidence to support that higher serum urate levels may cause lower cognitive function, higher systolic blood pressure, and higher risk of coronary heart disease and myocardial infarction. Further study in the form of clinical trials is required to explore such possible causal effects.

Contact Email: dipender.gill@imperial.ac.uk

\section{First analysis of prevalence and risk factors in South Asians migrated to the UK and Qatar compared with UK Caucasians: the Bio-Repository of
DNA in Stroke study (BRAINS)}

Taylor Aurelius, $B S c^{1}$, Dhanya Kumar, $B S c^{1}$, Thang S. Han, $M D$, $P h D^{1}$, Sapna Sharma, $M D^{1}$, Hasan Al Hail, $M D, F A A N^{2}$, Fahmi Khan, MBBS, CABMs's, and Pankaj Sharma, MD, $\mathrm{PhD}^{1,3}$; on behalf of the BRAINS Collaborators

Objectives Prior to a GWAS for ancestral stroke risk loci, we sought to compare the prevalence and risk factors between UKSA, QSA and UKC. Background Stroke incidence and mortality rates are declining but are ten-fold less in UK South Asians (UKSA) than UK Caucasians (UKC), and actually rising in Qatar (QSA) in the Middle East. Environmental and genetic factors play major roles but hitherto, there has been little research on stroke in patients of South Asian ancestry despite the UK hosting the largest congregation of this group outside Indian subcontinent. To gain insights into this discrepancy we established BRAINS, the World's largest South Asian stroke DNA repository. Design/Methods BRAINS is an ongoing global (UK, Middle East, India) repository of stroke in patients of South Asian and European ancestry. Comparisons of outcomes between the groups were made using chi-squared test. Results We recruited 1034 (639 males, 395 women) UKSA, 404 (330 males, 74 females) QSA and 1030 (590 males, 440 females) UKC with stroke. South Asians comprised 654 Indians (405 males, 249 females), 307 Pakistani (183 males, 124 females) and 73 Bangladeshis (51 males, 22 females). Compared with UKC, mean $( \pm \mathrm{SD})$ age of stroke onset was earlier in UKSA women $(65.4 \pm 16.1$ yrs vs $71.7 \pm 14.5 \mathrm{yrs}, p=0.030)$ and in UKSA men $(63.9 \pm 14.7$ yrs vs $67.9 \pm 13.4$ yrs, $p=0.016)$. Ischaemic stroke constituted the largest proportion of stroke subtype both for UKSA (82.3\%) and UKC (89.0\%) while haemorrhagic stroke 
occurred more frequently among UKSA than in UKC ( $13.8 \%$ vs $8.4 \%, p<0.001)$. Hypertension $(72.8 \%$ vs $65.2 \%, p=0.001)$ and type 2 diabetes $(47.1 \%$ vs $18.7 \%, p<0.001)$ were also more common in UKSA than in UKC. Among patients admitted with stroke and atrial fibrillation without previous treatment with an anticoagulant, only $25.0 \%$ of UKSA compared with $43.0 \%$ of UKC, were treated with this agent on discharge $(p=0.013)$. Conclusions Compared with indigenous UK population, UKSA had earlier onset and higher frequency of almost all major stroke risk factors. Genetic factors may play an important role in these individuals' susceptibility to stroke; we are now undertaking a South Asian stroke GWAS of BRAINS to identify specific population susceptibility risk loci.

Contact Email: Pankaj.Sharma@rhul.ac.uk

\section{What's in a name? Optimizing phenotype definition for ischemic stroke genetic discovery}

J. von Berg, $M S c^{1}$, S.W. van der Laan, $P h D^{2}$, J. de Ridder, $P h D^{1}$, B.D. Mitchell, $P h D, M P H^{3}$, S.J. Kittner, $M D, M P H^{3}$, and S.L. Pulit, $P h D^{1,3,4}$; on behalf of SiGN

Objective To improve genetic discovery in ischemic stroke (IS) by identifying more genetically-homogeneous diagnostic criteria while maintaining sufficient sample size. Background The TOAST and CCS classification systems have been developed to classify IS patients into cardioembolic stroke (CES), large artery stroke (LAS), and small vessel stroke (SVS). Previous work indicates that TOAST and CCS subtypes have moderate concordance and place over a third of all samples into an "undetermined" category. Design/Methods We used the SiGN dataset (13,930 IS cases and 28,026 controls) with TOAST, CCSc and CCSp subtypes. We define two new phenotypes: (1) intersect, for which an individual must be assigned the same subtype by all three systems and (2) union, for which an individual must be assigned a subtype by at least one of the systems. The union yields the largest sample size, thereby potentially improving power, while the intersect may be more genetically homogeneous (containing cases that share the same genetic risk modifiers), which can also improve power to detect new loci. The sample sizes for union are 2.0, 2.6 and 2.3 times larger than the intersect for CES, LAS, and SVS respectively. Results Heritability $\left(h^{2}\right)$ estimates show that the intersect yields a higher $h^{2}$ in all subtypes, suggesting genetic variation explains more of the variation in the intersect. The odds ratios (ORs) obtained for the intersect definition are in general, compared to the other phenotype definitions, more extreme at known stroke-associated loci. Using intersect, we find a SNP to be associated to SVS $(\mathrm{rs} 10029218$, OR $=1.27, p=1.2 \mathrm{e}-8)$ that is in the CAMK2D locus: a suggestive association in MEGASTROKE (rs10017904, OR $=1.17, p=7.2 \mathrm{e}-7$ ). Conclusions Here we show that using the intersect of the subtyping methods creates a more geneticallyhomogeneous trait definition yielding increased heritability estimates, that provides increased power to replicate known hits despite its lower sample size (figure 3).

Contact Email: skittner@som.umaryland.edu

\section{Causal associations of blood lipids with risk of ischaemic stroke and intracerebral haemorrhage}

Luanluan Sun, $P h D^{1,2}$, Robert Clarke, MD, FRCP, FFPH, FFPHI, $M S c, D C H^{1}$, Derrick Bennett, BSc, $M S c, P h D^{1}, Y u$ Guo, $P h D^{3}$, Robin Walters, $P h D^{1}$, Michael Hill, DPhil ${ }^{1,4}$, Sarah Parish, DPhil, MSc, BSc ${ }^{1,4}$, Iona Millwood, DPhil ${ }^{1,4}$, Zheng Bian, $M D, M S c^{3}$, Yiping Chen, MBBS, DPhil ${ }^{1,4}$, Canqing $Y u, P h D^{5}$, Jun $L v, P h D^{5}$, Rory Collins, FRS, FMedSci, Junshi Chen, $M D$, $\mathrm{PhD}^{6}$, Richard Peto, FRS ${ }^{1}$, Liming Li, MD, $\mathrm{MPH}^{3}$, and Zhengming Chen, DPhil ${ }^{1}$; on behalf of the China Kadoorie Biobank Collaborative Group

Objective Review ongoing LDL-C research and recently published results in Nature Medicine, 25, 569-74 (2019). doi: 10.1038/s41591-019-0366-x. Background Stroke is the second leading cause of death worldwide and accounts for $>2$ million deaths annually in China. Ischemic stroke (IS) and intracerebral hemorrhage (ICH) account for an equal number of deaths in China, despite a fourfold greater

Figure 3 (Abstract 23) Intersection of subtyping methods provides increased power to replicate known hits in the setting of smaller sample sizes
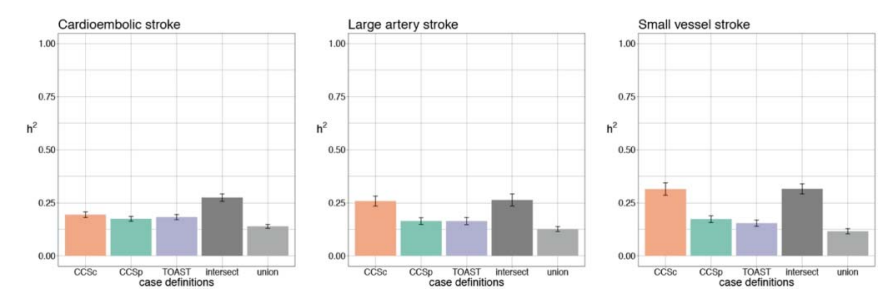
incidence of IS. Stroke incidence and ICH proportion are higher in China than in Western populations, despite having a lower mean low-density lipoprotein cholesterol (LDL-C) concentration. Observational studies reported weaker positive associations of LDL-C with IS than with coronary heart disease (CHD), but LDL-C-lowering trials demonstrated similar risk reductions for IS and CHD. Mendelian randomization studies of LDL-C and IS have reported conflicting results and concerns about the excess risks of ICH associated with lowering LDL-C may have prevented the more widespread use of statins in China. Design/Methods We examined the associations of biochemically measured lipids with stroke in a nested casecontrol study in the China Kadoorie Biobank (CKB) and compared the risks for both stroke types associated with equivalent differences in LDL-C in Mendelian randomization analyses. Results The results demonstrated positive associations of LDL-C with IS and equally strong inverse associations with $\mathrm{ICH}$, which were confirmed by genetic analyses and LDL-C-lowering trials. Conclusions Lowering LDL-C is still likely to have net benefit for the prevention of overall stroke and cardiovascular disease in China.

Contact Email: zhengming.chen@ndph.ox.ac.uk

\section{A genome-wide association study of white matter hyperintensities in 42,000 individuals}

\author{
E. Persyn, $P h D^{1}$, C.M. Lewis, $P h D^{1,2}$, M. Traylor, $P h D^{3}$, and \\ H.S. Markus, $M D^{3}$
}

Objective To investigate genetic factors of small vessel disease in UK Biobank imaging-derived phenotypes. Background White matter hyperintensities (WMH) confer increased risk of stroke and dementia. More diffuse white matter damage can be detected using diffusion tensor imaging (DTI), which has demonstrated abnormalities throughout the white matter in patients with cerebral small vessel disease. The genetic study of WMH and DTI white matter abnormalities may provide insights into the underlying neurobiology, and identify much-needed potential treatment targets. Design/Methods We performed a genome-wide association study (GWAS) in European UK Biobank samples on WMH $(\mathrm{n}=18,381)$, and on two DTI-derived markers (Fractional Anisotropy $[F A, n=17,663]$ and Mean Diffusivity $[M D, n=17,467]$ ). $\mathrm{FA}$ and $\mathrm{MD}$ phenotypes were derived from principal component analysis of regional values obtained using track based spatial statistics on 48 tracts. WMH GWAS results were meta-analysed with two multi-ethnic independent studies, from the CHARGE consortium (Verhaaren, 2015, $\mathrm{n}=21,079$ ) and in stroke patients (Traylor, $2019, \mathrm{n}=2,850)$, for a total of 42,310 individuals.
Transcriptome-wide association studies (TWAS) were conducted on GWAS summary statistics to identify genes with significant expression-trait association. Results We identified 18 significantly associated loci for WMH, 8 for FA and 6 for MD, of which 9 loci in WMH, 1 each in FA and $\mathrm{MD}$ were previously reported. There was a significant genetic correlation between the traits $(\mathrm{WMH} / \mathrm{FA}: \mathrm{rg}=$ -0.25 [0.06], $p=3.2 \mathrm{e}-05 ; \mathrm{WMH} / \mathrm{MD}: \mathrm{rg}=0.41$ [0.08], $p$ $=8.7 \mathrm{e}-08 ; \mathrm{FA} / \mathrm{MD}: \mathrm{rg}=-0.77[0.03], p=2.7 \mathrm{e}-114)$. TWAS identified 12 gene expression-trait associations in brain tissue from the CommonMind Consortium study for WMH, 3 for FA and 4 for MD. Conclusions In this large GWAS of WMH $(\mathrm{n}=42,310)$ and in GWAS of DTI parameters $(n>17,000)$, we identified novel loci for white matter damage, which will provide insights into the biological underpinnings.

Contact Email: hsm32@cam.ac.uk

\section{Genetic analysis for monogenic disorders in young age stroke patients: Results from the GENE_YAS study of the CRCS-K}

Keon-Joo Lee, MD, $\mathrm{MSc}^{1}$, Hong-Kyun Park, MD, MSc ${ }^{2}$, Joon Seol $\mathrm{Bae}, \mathrm{PhD}^{3}$, and Hee-Joon Bae, $M D, P h D^{1}$; on behalf of CRCS-K Investigators

Objective To evaluate the prevalence of 14 monogenic disorders associated with stroke, and to develop algorithms for screening monogenic disorders in young stroke patients. Background Although monogenic disorders are known to be associated especially with young stroke patients, they might have been underdiagnosed. Identifying genetic abnormalities in stroke would be important both for patients themselves and family members for preventing future stroke, and pre-symptomatic counseling. However, there had been no large prospective study with screening flow regarding their prevalence in stroke population. Design/Methods GENE_YAS is an ongoing study based on a prospective nationwide multicenter stroke registry (Clinical Research Collaboration for Stroke in Korea, CRCS-K). Among the consecutively enrolled patients, all patients aged 55 years or younger are eligible for this study except those who refuse to give consent. Genetic analysis using a New Generation Sequencing method will be performed to evaluate the prevalence of the 14 monogenic disorders. Clinical outcomes including 3-month and 1-year modified Ranking scale, and stroke recurrence, myocardial infarction, vascular death and all-cause death up to one year will be collected. The final sample size is planned to be 4,500. The figure 4 presents the study scheme. Results Until January 2019, a total of 198 patients (54\%) gave their consent to this study among 369 stroke patients aged less than or equal to 55 years and were registered into the CRCS-K registry. The Pilot analysis for 
Figure 4 (Abstract 26) Study schema of the GENE_YAS study, a prospective nationwide Korean multicenter stroke registry

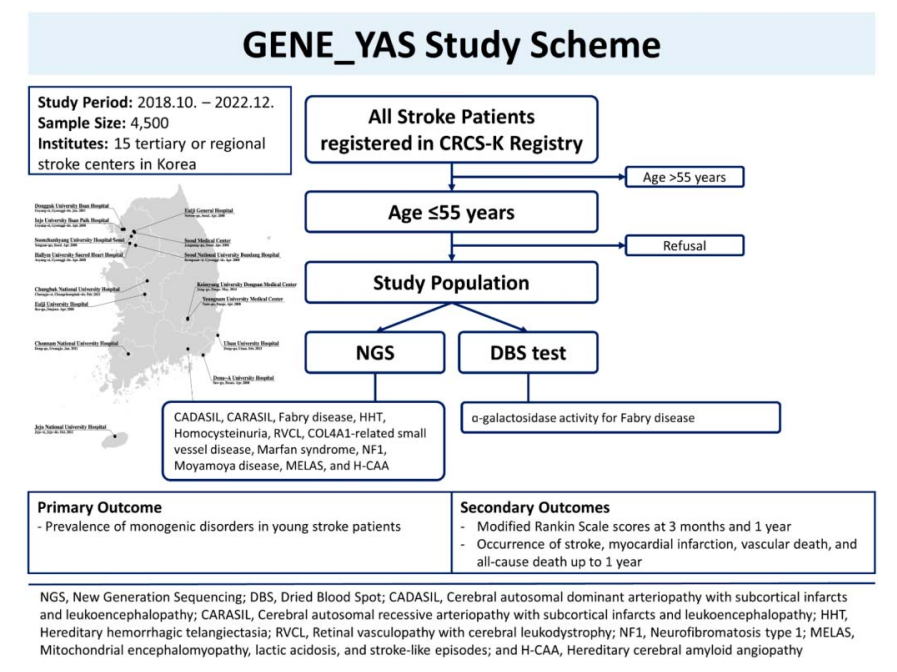

the first 84 patients did not reveal any pathogenic mutations yet. Conclusions We hope that this nationwide prospective large-scale genetic study can provide the prevalence of the major monogenic disorders in young-age Korean stroke population and information for their stroke characteristics and clinical outcomes.

Contact Email: gooday19@gmail.com 


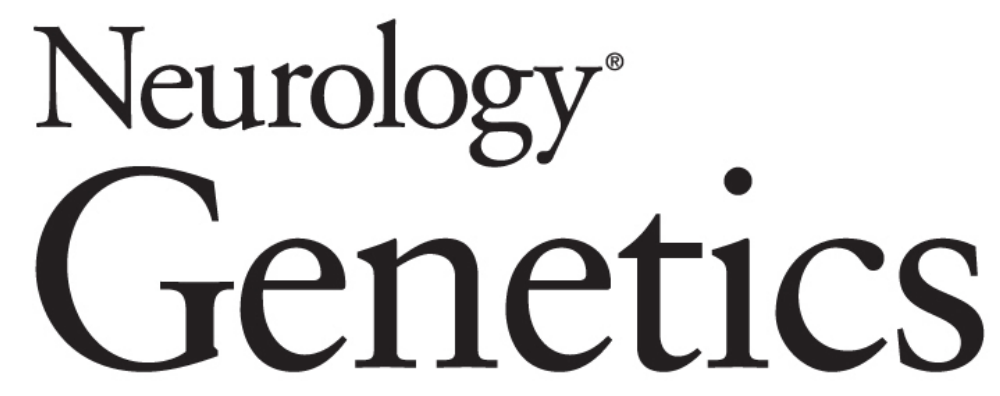

\section{Proceedings of the 25th International Stroke Genetics Consortium Workshop: Cambridge, UK}

Jemma C. Hopewell, Matthew Traylor and Hugh S. Markus

Neurol Genet 2020;6;e421

DOI 10.1212/NXG.0000000000000421

This information is current as of May 21, 2020

Updated Information \& Services

Permissions \& Licensing

Reprints including high resolution figures, can be found at: http://ng.neurology.org/content/6/1_Supplement_1/e421.full.html

Information about reproducing this article in parts (figures,tables) or in its entirety can be found online at:

http://ng.neurology.org/misc/about.xhtml\#permissions

Information about ordering reprints can be found online: http://ng.neurology.org/misc/addir.xhtml\#reprintsus

Neurol Genet is an official journal of the American Academy of Neurology. Published since April 2015, it is an open-access, online-only, continuous publication journal. Copyright $(2020$ American Academy of Neurology. All rights reserved. Online ISSN: 2376-7839.

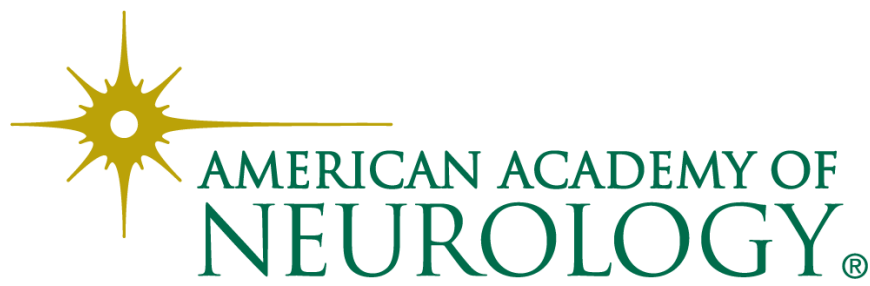

